\title{
TUGENDHAT: a pilot randomized study on effects of biventricular pacing in patients with bradycardia pacing indication and normal systolic function on heart failure, atrial fibrillation and quality of life (results of 12 month follow-up)
}

\author{
Taborsky $\mathrm{M}^{1}$, Rihova $\mathrm{D}^{2}$, Mraz $\mathrm{T}^{2}$, Mandysova $\mathrm{E}^{2}$, Vlasinova $\mathrm{J}^{5}$, Kamenik $\mathrm{L}^{3}$, Novak $\mathrm{M}^{6}$, Neuzil $\mathrm{P}^{2}$, \\ Jarkovsky $\mathrm{J}^{4}$, Littnerova $\mathrm{S}^{4}$
}

Department of Internal Medicine I - Cardiology, Faculty of Medicine and Dentistry, Palacky University Olomouc, Czech Republic. milos.taborsky@seznam.cz

\begin{abstract}
Background: Since the late 1990s, a growing number of clinical studies have indicated that long-term permanent right ventricular (RV) apical pacing will induce severe complications such as development of heart failure, increased burden of atrial fibrillation leading to decreased quality of life.

Aim of the study: To investigate whether cardiac resynchronization therapy (CRT) using biventricular (BiV) pacing can prevent the development of left ventricular (LV) dysfunction, LV remodelling, worsening of the clinical status and quality of life in chronically RV paced patients with normal LV ejection fraction (EF).

Methods and results: A total of 127 patients with Class I indication for permanent cardiac pacing and without established indication for CRT were subjected to 6 months of RV and BiV pacing in a patient-blinded, randomized crossover trial. Treatment effects of BiV pacing were evaluated for LV function, LV remodelling and clinical status. As compared with RV pacing, BiV pacing did not significantly prevent the decrease of LV function [LVEF 61.0\% (36.0; $68.0)$ vs $60.5 \%(38.5 ; 67.5)$ in RV pacing], did not change the functional class according to the New York Heart Association [52\% in Class II vs $53.9 \%$ in Class II in RV pacing, and $3.9 \%$ in Class III vs $6.9 \%$ in Class III in RV pacing], and did not present any changes in quality of life $[32.5(18.0 ; 80.0)$ vs $32.0(21.0 ; 47.0)$ indexes in RV pacing]. Conclusion: BiV pacing, compared to RV pacing, did not change LV function and quality of life in patients with the absence of LV dysfunction or remodelling, standard bradycardia pacing indications in a pilot phase (12month follow-up) of the TUGENDHAT trial. The final report will be published after 60 -month follow-up termination (Tab. 5, Fig. 3, Ref. 30). Full Text in PDF www.elis.sk.

Key words: BiV and RV pacing, LV remodelling, heart failure, atrial fibrillation, quality of life.
\end{abstract}

Results of several trials have supported the fact that right ventricular (RV) apical pacing might lead to adverse clinical outcomes in patients with standard pacing indications. Nevertheless, RV apical pacing continues to be practiced by many physicians because of its easy accessibility and relative stability over time $(1,2)$. The optimal mode and site of pacing remain undefined.

The detrimental effect of RV pacing is probably most important in patients with pre-existing left ventricular (LV) dysfunction and may lead to worsening of heart failure $(3,4)$. In RV pacing, the sequence of electrical activation resembles the activation

${ }^{1}$ Department of Internal Medicine I - Cardiology, Faculty of Medicine
and Dentistry, Palacky University Olomouc, Czech Republic, ${ }^{2}$ Cardio-
logy Department, Na Homolce Hospital, Prague, Czech Republic, ${ }^{3}$ Cardio-
logy Clinic, Central Military Hospital, Prague, Czech Republic, ${ }^{4}$ Institute
of Biostatistics and Analyses, Masaryk University, Brno, Czech Republic,
${ }^{5} 1$ st Internal - Cardiology Clinic, Masaryk University, Brno-Bohunice,
Czech Republic, and ${ }^{6} 1$ st Internal Cardiology - Angiology Clinic, Masaryk
University, Brno, St. Anna Hospital, Czech Republic Address for correspondence: M. Taborsky, MD, PhD, FESC, MBA, Department of Internal Medicine I - Cardiology, Faculty of Medicine and Dentistry, Palacky University Olomouc, Czech Republic, I.P. Pavlova 6, CZ-775 20 Olomouc, Czech Republic. pattern in left bundle branch block (LBBB). This asynchronous electrical pattern is accompanied by abnormal dyssynchronous mechanical interactions within the LV (5). Experimental data suggested that biventricular (BiV) pacing might preserve myocardial performance better than RV apical pacing in patients with atrioventricular (AV) block and normal systolic function (6). The underlying mechanism may be a significant reduction in LV systolic dyssynchrony, as shown by Cojoc et al (7). In the majority of patients with severe LV dysfunction and severe clinical heart failure associated with either LBBB- or RV pacing-induced dyssynchrony, BiV pacing improves clinical status, reduces mortality and morbidity, reduces heart failure (HF) hospitalizations, reverses LV remodelling and improves LV function $(8,9)$. Therefore, BiV pacing is nowadays recommended in patients with ventricular dyssynchrony (QRS $>120 \mathrm{~ms})$, severe LV dysfunction [LV ejection fraction $(\mathrm{LVEF})<0.35]$ and mild to severe clinical manifestation of HF [New York Heart Association (NYHA) classification II-IV] (10). In contrast, patients with pre-existing LV dysfunction and a Class I indication for standard pacing (QRS $<120 \mathrm{~ms}$ ) have improved LV systolic function, exercise capacity, and quality of life after BiV pacing as compared 


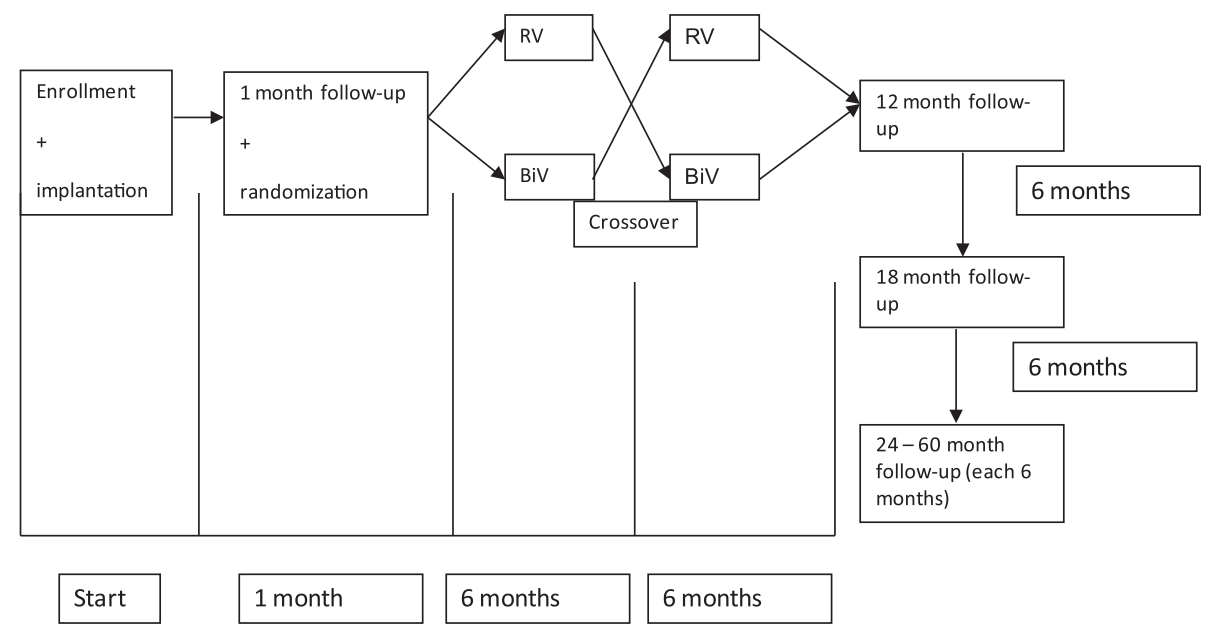

Fig. 1. Project Schedule.

with RV apical pacing (11). These promising data suggest that BiV pacing may be a feasible option for permanent pacing in the majority of patients who have normal LV systolic function and that it may prevent the adverse effect of conventional RV apical pacing on LV systolic function and other complications of longterm RV pacing.

\section{Methods}

\section{Study protocol}

TUGENDHAT (Trial on effect of biventricular pacing in patients with bradycardia and normal ejection fraction, with the aim of decreasing HF, atrial fibrillation and preventing complications) was a multicentric, prospective, randomized, single-blinded crossover comparison between RV and BV pacing.

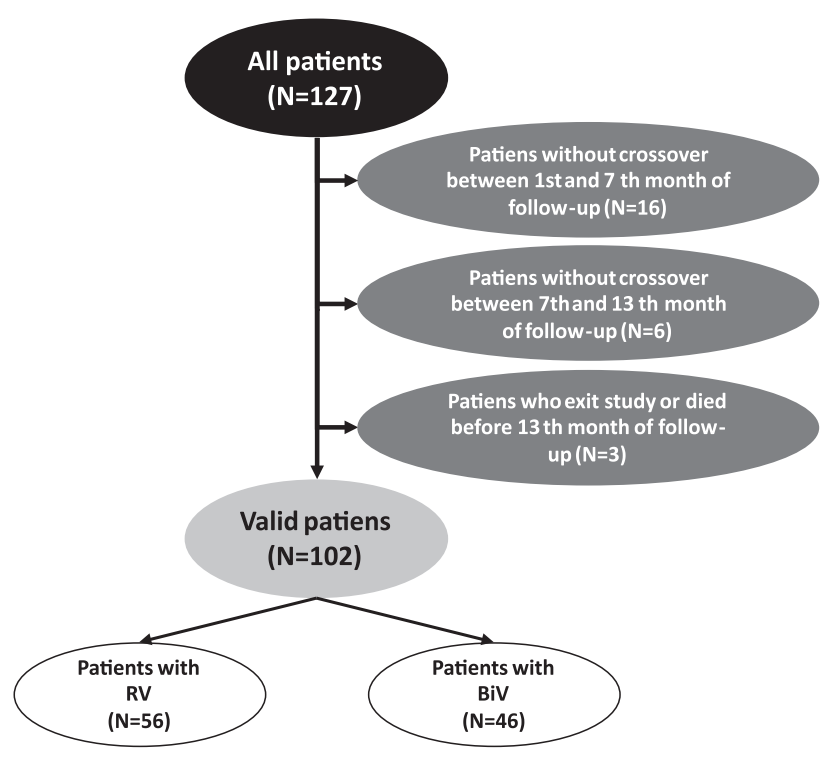

Fig. 2. Definition of Dataset.
It was an investigator-driven trial supported by IGA grant $\mathrm{Nr}$ NR9190-3/2007. A flow chart of the study protocol is shown in Figure 1. A flow chart of the study protocol is shown in Figure 1. A 1-month run-in phase was used to detect and correct any device- or lead-related dysfunctions before patients entered the randomization phase. After 1:1 randomization to one of the two pacing modes ( $\mathrm{RV}$ or $\mathrm{BiV}$ ), the patients were followed for two 6-month study periods with crossover to the complementary pacing mode after the first 6-month interval. After this pilot phase, the patient population will be followed for a total of 5 years. In patients without atrial fibrillation (AF), the programmed AV delay was optimized at the beginning of each study period using echocardiography. Outcome measures were assessed at the end of each 6-month period. The study had three primary end points: (i) echocardiographic parameters [LVEF, left ventricular end-diastolic diameter (LVEDD), left ventricular end-systolic diameter (LVESD), mitral regurgitation and others], (ii) functional class according to the NYHA, and (iii) quality of life as assessed with the Minnesota Living with Heart Failure (MLHF) questionnaire. (12) Serum concentration of Nterminal pro-B-type natriuretic peptide (NT-proBNP), AF burden, pacing parameters and complications of pacing were classified as secondary end points.

\section{Study population}

A total of 127 patients with documented symptomatic bradycardia, Class I indications for permanent pacing and preserved LV function (LVEF $>0.45$ ) were enrolled in the study. These indications included sinus node dysfunction and bradycardia due to advanced AV block. Chronic medication should be maintained throughout the study period and any changes will be documented. After an absence of 22 crossovers ( 16 from RV to BiV and 6 from $\mathrm{BiV}$ to RV) and 3 deaths during the study, complete data sets were available for 102 patients (Fig. 2). Baseline characteristics of these patients are presented in Tables 1 and 2. The study was approved by the local ethics committee. All patients gave informed consent before device implantation. 
Tab. 1. Characteristics of patients before device implantation.

\begin{tabular}{lc}
\hline & Description $^{1}$ \\
\hline Gender (Female) & $\mathrm{N}=39(38.2 \%)$ \\
Age at device implant (yrs.) & $72.0(57.0 ; 83.0)$ \\
Subject's height (cm) & $171.0(155.0 ; 186.0)$ \\
Subject's weight (kg) & $80.0(62.0 ; 110.0)$ \\
Body-Mass Index & $27.1(22.5 ; 35.2)$ \\
Quality of Live (MLFH index) & $31.5(18.0 ; 47.0)$ \\
Hearth rhythm history & \\
$\quad$ Sinus rhythm & $\mathrm{n}=80(78.4 \%)$ \\
$\quad$ Atrial fibrillation & $\mathrm{n}=21(20.6 \%)$ \\
$\quad$ Atrial flutter & $\mathrm{n}=1(1.0 \%)$ \\
Other & $\mathrm{n}=0(0.0 \%)$ \\
NYHA functional classification & \\
$\quad$ Class I & $\mathrm{n}=0(0.0 \%)$ \\
$\quad$ Class II & $\mathrm{n}=14(13.7 \%)$ \\
$\quad$ Class IV $I V$ & $\mathrm{n}=88(86.3 \%)$ \\
Ejection fraction (\%) & $\mathrm{n}=0(0.0 \%)$ \\
${ }^{1}$ median supplemented by 5th-95th percentile for continuous parameters; number of \\
patients and percentage of category for categorical variables
\end{tabular}

\section{Device implantation}

All patients enrolled in the study received biventricular pacemakers delivering either RV pacing or BiV pacing, depending on the programming of the device (InSync III, Medtronic, Inc.), using the 2090 programmer and 9998 software (Medtronic, Inc.). The right atrial and RV leads were positioned at the right atrial appendage and the RV leads at the RV apex (41) or mid-septum (61). The LV leads were introduced transvenously through the coronary sinus venous branch and placed in the lateral, posterolateral or poste- rior vein. The lead position was documented by X-ray in the AP, LAO 30 deg., and RAO 30 deg. projections. Successful LV lead implantation was performed in 116 out of the 127 patients (91\%).

\section{Echocardiographic evaluation}

All measurements were performed according to the guidelines of the European Society of Echocardiography using M-mode recordings for unidimensional parameters and Simpson's biplane method for LV volumes. The LV hypertrophy index was calculated as the sum of LV end-diastolic septal and posterior wall thickness divided by end-diastolic diameter and given as a percentage. Left and right ventricular heart cycle intervals were measured by pulsed Doppler echocardiography. Left ventricular dp/dt was estimated from mitral regurgitation flow. Echocardiography was performed using the Vivid 7 ultrasound system (GE Medical Systems). Echocardiographic data were stored on magneto-optical hard disks and analyzed offline with the EchoPac workstation (GE Medical Systems) using the average value of 8 consecutive measurements for each parameter.

\section{Quality of life assessment, NT-proBNP measurements}

Quality of life assessment was performed with the 36-Item Short-Form General Health Survey (SF-36) before implant and during follow-up visits at 6 and 12 months, and the assessment will be repeated every 12 months until the end of the study.

Venous blood samples were taken after 1-hour rest at the beginning of each follow-up visit. The samples were centrifuged within 1 hour and stored at $-60^{\circ} \mathrm{C}$ for a maximum of 4 weeks. Serum concentrations of NT-proBNP were measured using an electrochemiluminescence immunoassay (Roche Diagnostics).

Tab. 2. Diagnosis of patients according to randomized groups. Patient s may have multiple diagnoses.

\begin{tabular}{|c|c|c|c|c|c|c|}
\hline \multirow[b]{2}{*}{ Sinus Arrhythmias } & \multicolumn{2}{|c|}{$\begin{array}{c}\text { Total } \\
102\end{array}$} & \multicolumn{2}{|c|}{$\begin{array}{c}\text { RV } \\
56\end{array}$} & \multicolumn{2}{|c|}{$\begin{array}{c}\mathrm{BiV} \\
46\end{array}$} \\
\hline & 80 & $78.4 \%$ & 46 & $82.1 \%$ & 34 & $73.9 \%$ \\
\hline Sinus arrest/pause/exit block & 1 & $1.0 \%$ & - & - & 1 & $2.2 \%$ \\
\hline Sinus bradycardia & 29 & $28.4 \%$ & 16 & $28.6 \%$ & 13 & $28.3 \%$ \\
\hline Sinus tachycardia & 1 & $1.0 \%$ & - & - & 1 & $2.2 \%$ \\
\hline Brady-tachy syndrome & 5 & $4.9 \%$ & 3 & $5.4 \%$ & 2 & $4.3 \%$ \\
\hline Sick sinus syndrome & 57 & $55.9 \%$ & 32 & $57.1 \%$ & 25 & $54.3 \%$ \\
\hline Other, specify & 1 & $1.0 \%$ & 1 & $1.8 \%$ & - & - \\
\hline Atrial Arrhythmias & 47 & $46.1 \%$ & 26 & $46.4 \%$ & 21 & $45.7 \%$ \\
\hline Paroxysmal atrial fibrillation & 34 & $33.3 \%$ & 16 & $28.6 \%$ & 18 & $39.1 \%$ \\
\hline Persistent atrial fibrillation & 3 & $2.9 \%$ & 2 & $3.6 \%$ & 1 & $2.2 \%$ \\
\hline Permanent atrial fibrillation & 4 & $3.9 \%$ & 3 & $5.4 \%$ & 1 & $2.2 \%$ \\
\hline Atrial flutter & 12 & $11.8 \%$ & 9 & $16.1 \%$ & 3 & $6.5 \%$ \\
\hline Atrial tachycardia & 2 & $2.0 \%$ & 1 & $1.8 \%$ & 1 & $2.2 \%$ \\
\hline Ventricular Arrhythmias & 1 & $1.0 \%$ & 1 & $1.8 \%$ & - & - \\
\hline Non-sustained ventricular tachycardia & - & - & 1 & $1.8 \%$ & - & - \\
\hline AV Blocks and conduction disturbance & 33 & $32.4 \%$ & 17 & $30.4 \%$ & 16 & $34.8 \%$ \\
\hline 1st degree AV block & 12 & $11.8 \%$ & 7 & $12.5 \%$ & 5 & $10.9 \%$ \\
\hline 2nd degree AV block, Type I (Wenckebach) & 2 & $2.0 \%$ & 2 & $3.6 \%$ & - & - \\
\hline 2nd degree AV block, Type II(Mobitz) & 14 & $13.7 \%$ & 7 & $12.5 \%$ & 7 & $15.2 \%$ \\
\hline Intermitent 3rd degree AV block & 8 & $7.8 \%$ & 3 & $5.4 \%$ & 5 & $10.9 \%$ \\
\hline Permanent 3rd degree AV block & 3 & $2.9 \%$ & 1 & $1.8 \%$ & 2 & $4.3 \%$ \\
\hline Other major diseases & 38 & $37.3 \%$ & 19 & $33.9 \%$ & 19 & $41.3 \%$ \\
\hline Diabetes & 24 & $23.5 \%$ & 13 & $23.2 \%$ & 11 & $23.9 \%$ \\
\hline Hypertension & 4 & $3.9 \%$ & 2 & $3.6 \%$ & 2 & $4.3 \%$ \\
\hline Hypo/hyperthyroidism & 10 & $9.8 \%$ & 5 & $8.9 \%$ & 5 & $10.9 \%$ \\
\hline Other & 4 & $3.9 \%$ & 2 & $3.6 \%$ & 2 & $4.3 \%$ \\
\hline
\end{tabular}


Tab. 3. Differences between baseline, RV and BV in Echocardiographic Parameters.

\begin{tabular}{|c|c|c|c|c|c|c|}
\hline $\mathrm{n}=102$ & Baseline $^{1}$ & Right ventricular ${ }^{1}$ & Biventricular $^{1}$ & BL vs. RV ${ }^{2}$ & BL vs. Biv $^{2}$ & RV vs.Biv ${ }^{2}$ \\
\hline R-R interval (ms) & $907(638 ; 1250)$ & $850.0(695 ; 996)$ & $878(666 ; 1000)$ & $* *$ & $*$ & N.S. \\
\hline LVEDD (mm) & $51.0(43.0 ; 60.0)$ & $50.0(43.0 ; 60.5)$ & $50.0(44.0 ; 61.0)$ & N.S. & N.S. & N.S. \\
\hline $\operatorname{LVESD}(\mathrm{mm})$ & $35.0(28.0 ; 47.0)$ & $35.5(28.0 ; 50.5)$ & $36.0(28.0 ; 51.0)$ & $* * *$ & ** & N.S. \\
\hline LVEDV (ml) & $115.0(80.0 ; 180.0)$ & $115.0(82.0 ; 195.0)$ & $110.0(83.0 ; 200.0)$ & N.S. & N.S. & N.S. \\
\hline LVESV (ml) & $43.0(28.0 ; 89.0)$ & $45.0(28.0 ; 113.0)$ & $42.5(28.0 ; 126.0)$ & $* * *$ & $* *$ & N.S. \\
\hline Ejection fraction $(\%)$ & $62.0(46.0 ; 72.0)$ & $60.5(38.5 ; 67.5)$ & $61.0(36.0 ; 68.0)$ & $* * *$ & $* * *$ & $*$ \\
\hline Left atrium diameter (mm) & $44.0(37.0 ; 52.0)$ & $46.0(37.0 ; 55.0)$ & $45.0(37.0 ; 56.0)$ & $* * *$ & $* *$ & N.S. \\
\hline LV max sP/dt (mm Hg/s) & $0.00(0.00 ; 1400.00)$ & $0.00(0.00 ; 1550.00)$ & $0.00(0.00 ; 1400.00)$ & N.S. & N.S. & N.S. \\
\hline LV Ejection time (ms) & $286.5(222.0 ; 341.0)$ & $282.0(237.5 ; 331.5)$ & $290.0(240.0 ; 344.0)$ & N.S. & N.S. & N.S. \\
\hline Left Preejection Interval (ms) & $108.5(84.0 ; 162.0)$ & $145.0(105.0 ; 190.0)$ & $140.0(105.0 ; 177.0)$ & $* * *$ & $* * *$ & N.S. \\
\hline Right Pre Ejection Interval (ms) & $109.5(85.0 ; 160.0)$ & $130.0(95.5 ; 185.0)$ & $125.0(95.0 ; 160.0)$ & $* * *$ & $* * *$ & $*$ \\
\hline Q-Lateral Wall Contraction (ms) & $100.0(70.0 ; 190.0)$ & $135.0(97.5 ; 237.5)$ & $135.0(100.0 ; 200.0)$ & $* * *$ & $* * *$ & N.S. \\
\hline Q-E Wave (ms) & $495.5(408.0 ; 625.0)$ & $530.0(442.5 ; 627.5)$ & $530.0(425.0 ; 626.0)$ & $* * *$ & $* * *$ & N.S. \\
\hline LV diastolic Filling (E-A) (ms) & $444.0(230.0 ; 745.0)$ & $402.5(230.0 ; 543.5)$ & $400.0(220.0 ; 570.0)$ & * & * & N.S. \\
\hline E-Wave Amplitude (cm/s) & $63.5(38.0 ; 140.0)$ & $63.5(40.0 ; 142.5)$ & $60.5(40.0 ; 140.0)$ & N.S. & N.S. & N.S. \\
\hline A-Wave amplitude $(\mathrm{cm} / \mathrm{s})$ & $71.0(0.0 ; 108.5)$ & $65.0(0.0 ; 104.0)$ & $65.0(0.0 ; 120.0)$ & * & N.S. & N.S. \\
\hline E-Wave Deceleration Time (ms) & $220.0(120.0 ; 360.0)$ & $200.0(128.5 ; 280.0)$ & $200.0(130.0 ; 300.0)$ & $* * *$ & $\begin{array}{r}* * \\
\text { N.S. }\end{array}$ & $\begin{array}{l}\text { N.S. } \\
\text { N.S. }\end{array}$ \\
\hline \multicolumn{7}{|l|}{ Mitral regurgitation } \\
\hline None & $20(19.6 \%)$ & $16(15.7 \%)$ & $12(11.8 \%)$ & N.S. & N.S. & N.S. \\
\hline Grade I & $74(72.5 \%)$ & $69(67.6 \%)$ & $77(75.5 \%)$ & N.S. & N.S. & N.S. \\
\hline Grade II & $8(7.8 \%)$ & $15(14.7 \%)$ & $7(6.9 \%)$ & N.S. & N.S. & $*$ \\
\hline Grade III & - & - & $2(2.0 \%)$ & - & N.S. & N.S. \\
\hline Grade I (ms) & $360.0(240.0 ; 470.0)$ & $380.0(320.0 ; 470.0)$ & $400.0(316.0 ; 476.0)$ & $* *$ & $* *$ & N.S. \\
\hline Grade II (ms) & $465.0(360.0 ; 500.0)$ & $420.0(273.0 ; 500.0)$ & $430.0(336.0 ; 520.0)$ & N.S. & N.S. & N.S. \\
\hline Grade III (ms) & - & - & $412.5(400.0 ; 425.0)$ & - & - & - \\
\hline Presystolic regurgitation & $4(3.9 \%)$ & $1(1.0 \%)$ & $1(1.0 \%)$ & N.S. & N.S. & N.S. \\
\hline Pulmonary Artery Diastolic Press. & $22(21.6 \%)$ & $19(18.6 \%)$ & $20(19.6 \%)$ & N.S. & N.S. & N.S. \\
\hline
\end{tabular}

Tab. 4. Differences between baseline, RV and BV pacing in patients characteristics, NYHA Classification and Quality of life.

\begin{tabular}{|c|c|c|c|c|c|c|}
\hline $\mathrm{n}=102$ & Baseline $^{1}$ & Right ventricular $^{1}$ & Biventricular $^{1}$ & BL vs. RV $^{2}$ & BL vs. $\mathrm{Biv}^{2}$ & RV vs.Biv ${ }^{2}$ \\
\hline Subject's weight (kg) & $80.0(62.0 ; 110.0)$ & $80.0(62.0 ; 110.0)$ & $80.0(62.0 ; 110.0)$ & N.S. & N.S. & N.S. \\
\hline Body-Mass Index & $27.1(22.5 ; 35.2)$ & $27.1(23.5 ; 34.8)$ & $27.1(23.5 ; 34.8)$ & N.S. & N.S. & N.S. \\
\hline \multicolumn{7}{|l|}{ Heart rhythm } \\
\hline Sinus rhythm & $\mathrm{n}=80(78.4 \%)$ & $\mathrm{n}=87(85.3 \%)$ & $\mathrm{n}=88(86.3 \%)$ & N.S. & * & N.S. \\
\hline Atrial fibrillation & $\mathrm{n}=21(20.6 \%)$ & $\mathrm{n}=14(13.7 \%)$ & $\mathrm{n}=14(13.7 \%)$ & N.S. & N.S. & N.S. \\
\hline Atrial flutter & $\mathrm{n}=1(1.0 \%)$ & $\mathrm{n}=1(1.0 \%)$ & - & N.S. & N.S. & N.S. \\
\hline \multicolumn{7}{|l|}{ Other } \\
\hline \multicolumn{7}{|l|}{ NYHA (class) } \\
\hline Class I & - & $\mathrm{n}=40(39.2 \%)$ & $\mathrm{n}=44(43.1 \%)$ & **** & **** & N.S. \\
\hline Class II & $\mathrm{n}=14(13.7 \%)$ & $\mathrm{n}=14(53.9 \%)$ & $\mathrm{n}=53(52.0 \%)$ & $* * *$ & $* * *$ & N.S. \\
\hline Class III & $\mathrm{n}=88(86.3 \%)$ & $\mathrm{n}=7(6.9 \%)$ & $\mathrm{n}=4(3.9 \%)$ & $* * *$ & $* * *$ & N.S. \\
\hline Class IV & - & - & $\mathrm{n}=1(1.0 \%)$ & & N.S. & N.S. \\
\hline Quality of Life (MLHF score) & $31.5(18.0 ; 47.0)$ & $32.0(21.0 ; 47.0)$ & $32.5(18.0 ; 50.0)$ & N.S. & N.S. & N.S. \\
\hline Resting heart rate (bpm) & $65.0(45.0 ; 88.0)$ & $68.0(60.0 ; 85.0)$ & $70.0(62.0 ; 80.0)$ & ** & ** & N.S. \\
\hline Respiratory rate (breaths per min.) & $16.0(14.0 ; 19.0)$ & $16.0(14.0 ; 18.0)$ & $16.0(14.0 ; 18.0)$ & N.S. & N.S. & N.S. \\
\hline \multicolumn{7}{|l|}{ Current blood pressure } \\
\hline Systolic ( $\mathrm{mm} \mathrm{Hg}$ ) & $143.5(120.0 ; 180.0)$ & $133.0(111.0 ; 170.0)$ & $135.0(110.0 ; 170.0)$ & $* * *$ & ** & N.S. \\
\hline Diastolic ( $\mathrm{mm} \mathrm{Hg}$ ) & $80.0(60.0 ; 95.0)$ & $80.0(65.0 ; 95.0)$ & $80.0(60.0 ; 95.0)$ & N.S. & N.S. & N.S. \\
\hline LV Ejection fraction (\%) & $62.0(46.0 ; 72.0)$ & $60.5(38.5 ; 67.5)$ & $61.0(36.0 ; 68.0)$ & $* * *$ & $* * *$ & * \\
\hline
\end{tabular}

${ }^{1}$ median supplemented by 5 th-95th percentile for continuous parameters; number of patients and percentage of category for categorical variables

${ }^{2}$ Differences between baseline, RV and BV pacing periods were assessed using Wilcoxon Signed Ranks Test, when parameters are continuous, and McNemar's test, for categorical parameters; N.S. $\mathrm{p}>0.05$ no statistical significance, ${ }^{* * *} \mathrm{p}<0.001, * * \mathrm{p}<0.01,{ }^{*} \mathrm{p}<0.05$

\section{Statistical analysis}

Standard descriptive statistics were used for the analysis. Continuous parameters such as age, weight and body mass index were described by the median and 5th and 95 th percentile. Categorical parameters were described by their count and percentages. Differences between baseline, $\mathrm{RV}$ and $\mathrm{BiV}$ pacing periods were assessed using the Wilcoxon signed-rank test when parameters were continuous and McNemar's test for categorical parameters. IBM 


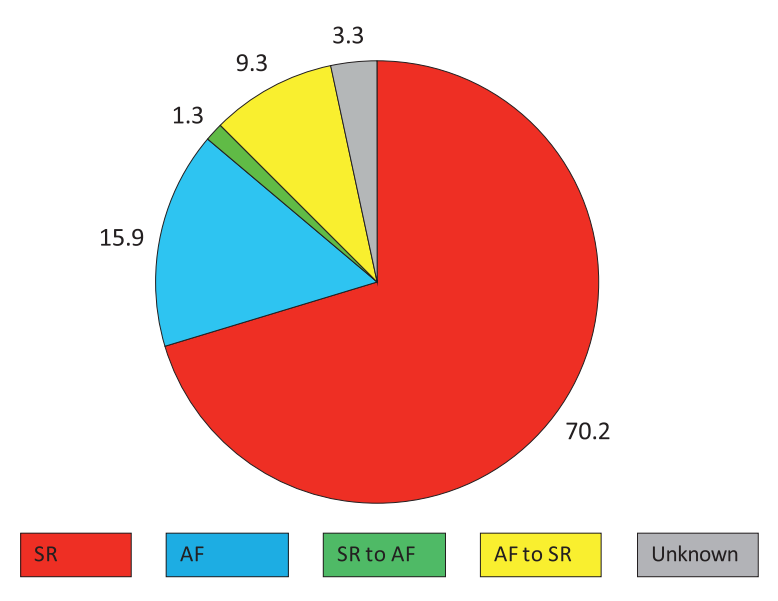

Fig. 3. Heart Rhythm at 12 month.

SPSS 19 for Windows (Release 19.0.1, IBM Corporation 2010) was used for data analysis.

\section{Results}

Study dropouts, complications of the therapy and optimization of drug therapy

There were no periprocedural deaths. Three of the 127 patients $(2.3 \%)$ died during the study, all after randomization to RV $(n=2)$ and BiV pacing $(n=1)$. The causes of death were fatal cerebral stroke, pulmonary embolism and malignancy. Because end points could only be analyzed in patients without missing data after completion of both crossover phases, all results are based on the 102 patients who completed the study.

Re-operations were required in 11 of these patients (10\%) due to dislodgement of the LV lead in 7 cases and due to bleeding into the pacemaker pocket in 4 cases.

Due to bradycardia, beta blockers were excluded before device implantation. One month thereafter, $82 \%$ of all patients were on medication with beta blockers and $86 \%$ on angiotensin-converting enzyme inhibitors or angiotensin II receptor blocker ther- apy. Drug therapy was not changed during the two crossover periods.

\section{Assessment of the primary end points}

In the pilot phase of the study (12-month follow-up, 6 months in $\mathrm{RV}$ and 6 months in BiV pacing), all 3 primary end points for the comparison between RV and BiV pacing were not met (Tabs 2 and 3 ). The most notable change after device implantation is the shift of patients from functional Class III to Classes I and II (more than $90 \%$ of patients are in NYHA I+II after device implantation). No statistically significant differences were found between the pacing modes. Similar results were also found for systolic blood pressure, resting heart rate and for BiV pacing mode also for increased presence of sinus rhythm (SR) (Tab. 3). Statistically significant changes after device implantation but without difference between the pacing modes were found for some echocardiographic parameters (Tab. 2). Statistically significant differences between the pacing modes were detected for RV pre-ejection interval, mitral regurgitation grade and ejection fraction (EF). Nevertheless, the real clinical significance of changes in LVEF and other measured parameters is questionable.

Quality of life measured by MLHF was 32 at study entry and no statistically significant changes were found after device implantation or between the pacing modes (Tab. 3). Analysis of subgroups divided according to various parameters was performed to find further differences in results achieved by RV and BiV pacing. No significant differences were, however, found in most of the parameters.

\section{Assessment of the secondary end points}

In terms of the secondary end points, NT-proBNP serum concentrations were not severely elevated in the study population before implant with a wide range for individual values during each of the two pacing periods. When compared with RV pacing, BiV stimulation did not reduce NT-proBNP concentration (Tab. 3). After 12 -month follow-up, where $70 \%$ of all patients were in SR and $15.3 \%$ in AF, we could observe a change from AF to SR in $8.7 \%$ of patients with no significant difference between the RV and $\mathrm{BiV}$ pacing modes (Fig. 3). Also the differences in standard

Tab. 5. Differences between baseline, RV and BV in Pacing Parameters.

\begin{tabular}{|c|c|c|c|c|c|c|}
\hline $\mathrm{n}=102$ & Baseline $^{1}$ & Right ventricular $^{1}$ & Biventricular $^{1}$ & BL vs. RV $^{2}$ & BL vs. $\mathrm{Biv}^{2}$ & RV vs.Biv ${ }^{2}$ \\
\hline \multicolumn{7}{|l|}{ Right atrium } \\
\hline P-potential $(\mathrm{mV})$ & $2.10(0.50 ; 4.80)$ & $2.30(0.30 ; 4.50)$ & $2.50(0.25 ; 1.00)$ & N.S. & N.S. & N.S. \\
\hline Threshold $(\mathrm{V} / \mathrm{mA})$ & $1.05(0.20 ; 1.70)$ & $2.50(2.00 ; 3.50)$ & $2.00(2.00 ; 3.00)$ & N.S. & N.S. & N.S. \\
\hline Resistance (Ohms) & $512.0(344.0 ; 760.0)$ & $652.0(278.0 ; 917.0)$ & $557.0(412.0 ; 860.0)$ & N.S. & N.S. & N.S. \\
\hline \multicolumn{7}{|l|}{ Right ventricle } \\
\hline$R$-potential $(\mathrm{mV})$ & $11.3(3.6 ; 22.4)$ & $10.6(6.00 ; 12.80)$ & $11.7(7.80 ; 18.00)$ & N.S & N.S. & N.S. \\
\hline Threshold (V/mA) & $0.80(0.20 ; 1.80)$ & $2.20(2.00 ; 3.00)$ & $2.00(1.20 ; 3.00)$ & N.S. & N.S. & N.S. \\
\hline Resistance (Ohms) & $649.0(495.0 ; 944.0)$ & $721.0(559.0 ; 987.0)$ & $664.0(3820 ; 877.0)$ & N.S. & N.S. & N.S. \\
\hline \multicolumn{7}{|l|}{ Left ventricle } \\
\hline$R$-potential $(\mathrm{m} / \mathrm{V})$ & $12.5(4.0 ; 28.5)$ & $11.60(5.40 ; 22.80)$ & $10.4(7.70 ; 15.00)$ & N.S. & N.S. & N.S. \\
\hline Threshold (V/mA) & $1.40(0.40 ; 5.00)$ & $1.70(2.00 ; 5.00)$ & $2.20(2.00 ; 5.00)$ & N.S. & N.S. & N.S. \\
\hline Resistance (Ohms) & $848.0(425.0 ; 1366.0)$ & $587.0(438.0 ; 762.0)$ & $559.0(449.0 ; 917.0)$ & N.S. & N.S. & N.S. \\
\hline
\end{tabular}

${ }^{1}$ median supplemented by 5 th-95th percentile for continuous parameters; number of patients and percentage of category for categorical variables

${ }^{2}$ Differences between baseline, RV and BV pacing periods were assessed using Wilcoxon Signed Ranks Test, when parameters are continuous, and McNemar's test, for categorical parameters; N.S. $\mathrm{p}>0.05$ not statistical significance, $* * * \mathrm{p}<0.001, * * \mathrm{p}<0.01,{ }^{*} \mathrm{p}<0$. 
pacing parameters and complications between the RV and BiV modes were not observed during the 12-month follow-up (Tab. 4).

\section{Discussion}

The TUGENDHAT trial is focused on the effects of RV and BiV pacing modes in patients with a conventional indication for permanent pacing (primary bradycardia indication) with normal LVEF.

The study shows that in a short follow-up period (12 months after the implant), RV apical pacing did not have a detrimental effect on LV systolic function in patients with normal EF and indications for pacing due to bradycardia. The adverse cardiac remodelling might be prevented by BiV pacing probably after a longer period of follow-up. Despite the use of RV apical pacing, development of HF, increased morbidity and mortality have been recognized in the past 10 years with the publication of the results of various large trials of pacemakers and implantable cardioverterdefibrillators $(13,14,15)$. The adverse clinical events seem to be related to a high cumulative percentage of RV apical pacing (16). This type of pacing causes an abnormal left ventricular electricalactivation sequence with an electromechanical delay in contraction (right to left ventricle dyssynchrony) and, subsequently, increased mitral regurgitation and decreased EF (17-20). Several preventive pacing algorithms have been developed to reduce the percentage of right ventricular pacing but these might be used only in patients with sinus node dysfunction, not in patients with advanced AV block and a high percentage of ventricular pacing $(21,22)$. In elderly patients with underlying risk factors for heart failure (diastolic dysfunction and comorbidities), more than $50 \%$ of RV apical pacing may trigger the complications (23).

The most apparent result of $\mathrm{RV}$ or $\mathrm{BiV}$ pacing in our trial is change in the NYHA classification. Most of the patients reported perceived improvement from NYHA Class III at the beginning of the study Class I and II. Improvement in the NYHA classification is in agreement with other studies, especially concerning the difference between the baseline and end of follow-up $(24,25)$. Difference between both pacing modes, however, was not statistically significant. Quality of life assessed by means of the standard MLHF questionnaire remained at the same level for both modes and differences between the baseline and end of follow-up were not statistically significant. This fact is surprising with respect to the significant NYHA improvement mentioned above, since most of the MLHF questions are directly or indirectly related to physical functioning and correlate with the NYHA and 6-Minute Walk Test (6MWT) (26). Nevertheless, results of other studies concerning quality of life are very variable and do not always follow the above assumption. Some studies reported only slight improvement in quality of life in both groups observed, but with no significant difference between them (27). It is also noteworthy that the observation periods in the pilot phase of our study were short (6+6 months).

The median EF decreased in comparison to the baseline. It is also to be noted that the difference is unlikely to be significant from the clinical point of view. The difference between the RV and BiV modes was found to be statistically significant as well. However, the importance of such a difference from the clinical point of view is probably also negligible. It is important to note that the initial median value of EF was $62.0 \%$ in patients included in our study, which is still within the value range for healthy individuals. Improvement of this parameter is therefore unlikely to occur. In other studies comparing RV and BiV pacing, the EF baselines were in an approximate range of $25-45 \%$, i.e. with a much greater space for improvement (28). On the other hand, trials including patients with normal EF reported results consistent with those seen in our study so far. For example in the PACE study (6), the EF decreased in patients with RV pacing and no significant change was found in the BiV pacing group, with the baseline being approximately $60 \%$. The authors further suggested that EF decreases rapidly in vulnerable patients and that these patients might benefit even more from BiV pacing. (6) No significant changes in EF were found in either of the observed groups in the PREVENT-HF trial (29). Decrease of EF in BiV patients was also found (30), which supports our conclusions that change of EF in relatively healthy patients is not a reliable end point for comparison of patients with various pacing modes.

Mitral regurgitation severity was classified into four grades and the proportion of patients in individual did not significantly differ when comparing the baseline and both treatment groups, with one exception (number of patients in Grade II BiV group). Comparison with other studies is difficult in this case because various evaluation approaches have been used. Nevertheless, slight to moderate improvements are usually stated, particularly in the Biv mode (25, $29)$. Other evaluation end points - pacing parameters - acted according to expectations and in agreement with published results.

\section{Study limitations}

There are several limitations to this study. It was limited to a relatively small number of patients and a short follow-up period in the pilot phase. However, the study was designed with adequate power to test in long-term follow-up for the expected differences between the two pacing modes with respect to the primary and secondary end points. Using a crossover design, placebo effects of study inclusion and pacemaker modes can be minimized. Furthermore, patients were blinded for pacemaker configurations.

The success rate for implantation of the biventricular-pacing system was $91 \%$, which is lower than that for conventional dualchamber pacing but similar to that for pacemakers implanted in patients with standard BiV indications. Randomized trials with larger samples, long-term folow-up, and sufficient power to evaluate clinical outcomes between these two pacing strategies are expected.

\section{Results}

We conclude that BiV pacing compared to RV pacing did not change LV function and quality of life in patients with the absence of LV dysfunction or remodelling, standard bradycardia pacing indications in a pilot phase (12- month follow-up) of the TUGENDHAT trial. Hence, we still believe that BiV pacing might be superior to RV pacing in long term follow-up in prevention of 
LV remodelling, heart failure manifestation and decreased $\mathrm{AF}$ burden. Following reports of the study will be published after 24 and 60 months of follow-up.

\section{References}

1. Sweeny MO, Hellkamp AS, Ellenbogen KA et al. Adverse effect of ventricular pacing on heart failure and atrial fibrillation among patients with normal baseline QRS duration in a clinical trial of pacemaker therapy for sinus node dysfunction. Circulation 2003; 107: 2932-2937.

2. Thambo JB, Bordachar $\mathbf{P}$, Garrigue $S$ et al. Detrimental ventricular remodelingin patients with congenital complete heart block and chronic right ventricular apical pacing. Circulation 2004; 110: 3766-3772.

3. Mizutani N, Waseda K, Itou T. Comparative study of the effect of pacing mode on the interaction of the right and left ventricles. J ArtifOrgans 2004; 7: 77- 82 .

4. Bordachar P, Garrigue S, Lafitte $S$ et al. Interventricular and intraleft ventricular electromechanical delays in right ventricular paced patiens with heart failure: implications for upgrading to biventricular stimulation. Heart 2003; 89: 1401-1405.

5. Riedlbauchová L, Kautzner J, Hatala R, Buckingham TA. Is right ventricular outflow tract pacing an alternative to left ventricular/biventricular pacing? PACE 2004; 27: 871-877.

6. Frias PA, Corvera JS, Schmarkey L et al.Evaluation of myocardial performancewith conventional single-site ventricularpacing and biventricular pacing in a canine model of atrioventricular block. J Cardiovasc Electrophysiol 2003; 14: 996-1000.

7. Cojoc A, Reeves JG, Schmarkey L et. Effects of single-site versus biventricularepicardial pacing on myocardial performance in an immature animal model of atrioventricular block. J Cardiovasc Electrophysiol 2006; 17: 884-889.

8. Cleland JG, Daubert JC, Erdmann E et al. The effect of cardiac resynchronization on morbidity and mortality in heart failure. N Engl J Med 2005; 352: 1539-1549.

9. Daubert JC, Leclercq C, Donal E, Mabo P. Cardiac resynchronisation therapy in heart failure: current status. Heart Fail Rev 2006; 11: 147-154.

10. Dickstein K, Vardas PE, Auricchio A et al. Focused Update of ESC guidelines on device therapy in heart failure. Eur Heart J; 2010:doi:10.1093/ eurheartj/ehq337.

11. Kindermann M, Hennen B, Jung $\mathbf{J}$ et al. Biventicularversus conventional right ventricular stimulation for patients with standard paging indication and left ventricular dysfunction: the Homburg Biventricular Pacing Evaluation (HOBIPACE). J Am Coll Cardiol 2006; 47: 1927-1937.

12. Rector TS, Kubo SH, Cohn JN. Patients' self-assessment of thein congestive heart failure. Part II: content, reliability and validity of a new measure, the Minnesota Living with Heart Failure questionnaire. Heart Fail 1987; 3: 198-209.

13. Wilkoff BL, Cook JR, Epstein AE et al. Dual-chamber pacing or ventricular backuppacing in patients with an implantace defibrillator: the Dual Chamber and VVIImplantable Defibrillator (DAVID) Trial. JAMA 2002; 288: 3115-3123.

14. Steinberg JS, Fischer A, Wang $P$ et al.The clinical implications of cumulative right ventricular pacing in the Multicenter Automatic Defibrillator Trial II. J Cardiovasc Electrophysiol 2005; 16: 359-365.
15. Sweeney JS, Prinzen FW. A new paradigm for physiologic ventricular pacing. J Am Coll Cardiol 2006; 47: 282-288.

16. Thackray SD, Witte KK, Nikitin NP et al. The prevalence of heart failure and asymptomatic left ventricular systolic dysfunction in a typical regional pacemaker population. Eur Heart J 2003; 24: 1143-1152.

17. Prinzen FW, Augustijn CH, Arts T et al. Redistribution ofmyocardial fiber strain and blood flow by asynchronous activation. Am J Physiol 1990; 259: H300-H308.

18. Nielsen JC, Kristensen L, Andersen HR et al. A randomized comparison of atrial and dual-chamber pacing in 177 consecutive patients with sick sinus syndrome: echocardiographic and clinical outcome. J Am Coll Cardiol 2003; 42: 614-623.

19. Vanderheyden M, Goethals M,Anguera I et al. Hemodynamic deterioration following radiofrequency ablation of the atrioventricular conduction system. Pacing Clin Electrophysiol 1997; 20: 2422-2428.

20. Nahlawi M, Waligora M, Spies SM et al. Left ventricular function during and after right ventricular pacing. J Am Coll Cardiol 2004; 44: 1883-1888.

21. Gillis AM, Purerfellner H, Israel CW et al. Reducing unnecessary right ventricularpacing with the managed ventricular pacing mode in patients with sinus node disease and AV block. Pacing Clin Electrophysiol 2006; 29: 697-705.

22. Sweeney MO, Bank AJ, Nsah E et al. Minimizing ventricular pacing to reduce atrial fibrillation in sinus-node disease. N Engl J Med 2007; 357: 1000-1008.

23. Sweeney MO, Hellkamp AS. Heartfailure during cardiac pacing. Circulation 2006; 113: 2082v8.

24. Martinelli M, De Siqueira S, Costa R et al. Conventional Versus Biventricular Pacing in Heart Failure and Bradyarrhythmia: The COMBAT Study. J Cardiac Fail 2010; 16: 293-300.

25. Leclercq C, Cazeau S, Lellouche D et al. Upgrading from single chamber right ventricular to biventricular pacing in permanently paced patients with worsening heart failure: The RD-CHF study. Pace-Pacing Clinl Electrophysioly 2007; 30: S23-S30.

26. Garin O, Ferrer M, Pont A et al. Disease-specific health-related quality of life questionnaires for heart failure: a systematic review with meta-analyses. Quality Life Res 2009; 18: 71-85.

27. Brignole M, Gammage M, Puggioni E et al. Comparative assessment of right, left, and biventricular pacing in patients with permanent atrial fibrillation. Eur Heart J 2005; 26: 712-722.

28. Ritter O, Koller M, Fey B et al. Progression of heart failure in right univentricular pacing compared to biventricular pacing. Intern J Cardiol 2006; 110: 359-365.

29. Stockburger M, Gomez-Doblas J, Lamas G et al. Preventing ventricular dysfunction in pacemaker patients without advanced heart failure: results from a multicentre international randomized trial (PREVENT-HF). Eur J Heart Fail 2011; 13: 633-641.

30. Ren X, Han Z, Wang $Y$ et al. A Clinical Comparison Between a New Dual-Chamber Pacing Mode-AAIsafeR and DDD Mode. Amer J Med Sci 2010; 339: 145-147.

Received June 23, 2011. Accepted January 27, 2013. 